\title{
Referéndum constitucional en Italia y plebiscito territorial en Brasil. Un enfoque sistémico de gobernanza
}

\section{Constitutional referendum in Italy and territorial plebiscite in Brazil. A Systemic Governance Approach}

\author{
Josep Pont Vidal \\ Universidade Federal do Pará (UFPA) \\ josevidal@ufpa.br
}

\begin{abstract}
RESUMEN
La gobernanza en sus diversas extensiones y fundamentada en los recursos teóricos habituales, presenta limitaciones operativas observacionales que se manifiestan en el direccionamiento político y administrativo, y que se ponen de manifiesto en la política y el territorio. La hipótesis que sostenemos es que la gobernanza en su plano operativo ha de sostenerse la observación y análisis de los cambios funcionales de la sociedad que se manifiestan en diversos planos. La idea de "gobernanza sistémica» posibilita la capacidad conceptual de las diversas operaciones de las áreas de conocimiento, y abre posibilidades operativas de integrar los sistemas administrativo y político con el territorio con el subjetivo. Ilustramos las limitaciones de la gobernanza política habitualmente utilizada con dos casos: el referéndum constitucional en Italia (2016) y un plebiscito territorial en Brasil (2011).
\end{abstract}

\section{PALABRAS CLAVE}

Teoría de sistemas autorreferenciales, funcionalismo-constructivista, sistema político-administrativo, territorio, gobernanza sistémica.

\begin{abstract}
Governance in its various extensions and based on the usual theoretical resources presents observational operational limitations that are manifested in the political and administrative direction, and which are revealed in politics and territory. The hypothesis we hold is that governance at its operational level must be sustained in the functional changes of society manifested in different planes. The idea of «systemic governance» enables the conceptual capacity of the various operations in the areas of knowledge and opens up operational possibilities of integrating the administrative and political systems with the territory with the subjective one. We illustrate the limitations of political governance usually used with two cases: the constitutional referendum in Italy (2016) and a territorial plebiscite in Brazil (2011).
\end{abstract}

\section{KEYWORDS}

Theory of self-reference systems, functionalist-constructivist, political and administrative system, territory, systemic governance.

\section{SUMARIO}

INTRODUCCIÓN. 1. FUNDAMENTOS TEÓRICO-CONCEPTUALES DE LA GOBERNANZA SISTÉMICA. 1.1. POLITICAY TERRITORIO. 1.2. DIRECCIONAMIENTO ESTATAL Y GOBERNANZA. 1.3. DIFERENCIACIÓN FUNCIONAL DE LA SOCIEDAD Y OPERACIONES. 1.3.1. Diferenciación funcional y «triple diferenciación». 2. GOBERNANZA SISTÉMICA: PREMISAS TEÓRICO-CONCEPTUALES CONFIGURADORAS. 
2.1. SISTEMA E INTEGRACIÓN. 3. GOBERNANZA POLÍTICA Y TERRITORIO: ITALIA Y BRASIL. 3.1. EL REFERÉNDUM CONSTITUCIONAL EN ITALIA. 3.1.1. Antecedentes. 3.1.2. Resultados. 3.1.3. Análisis sistémico. 3.2. PLEBISCITO SECESIONISTA EN BRASIL (REGIÓN AMAZÓNICA). 3.2.1. Antecendentes. 3.2.2. Resultados. 3.2.3. Análisis sistémico. 3.2.4. Unas conclusiones. REFERENCIAS BIBLIOGRÁFICAS.

\section{INTRODUCCIÓN}

La gobernanza es concebida como el instrumento en el que se depositan las expectativas para la gestión y el gobierno de las políticas públicas, los cambios de formas de intervención pública y como paradigma del Estado para la regulación del conflicto social, o en una expresión resumida: «todas las formas básicas de la coordinación de la acción» (Mayntz, 2009:45). En sus diversas definiciones aparecen de forma constante en las publicaciones tres nociones centrales, complejidad, dinámica y diversidad. La teoría moderna de sistemas -o teoría neosistémica- sintetiza, profundiza y modifica sensiblemente estas nociones, y las amplia con el concepto central de autoorganización.

En este trabajo asumimos como punto de partida conceptual la perspectiva neosistémica. En esta es posible, al menos, diferenciar dos paradojas o problemas centrales en torno a esta teoría, uno de carácter teórico y otro operativo-observacional.

Por un lado, la teoría sistémica de gobernanza de Niklas Luhmann se confronta con la nueva teoría de la gobernanza y con las nociones conceptuales de «autonomía» y «autodirección» de la sociedad (SeIbststeuerung) (Willke, 2014; Mayntz; Scharpf, 1995), «self-governance» (Kooiman, 2003:92), «interactive governance» (Kooiman, et al. 2008) ${ }^{1}$, «network governance» (Manna, 2010), y «evolutionary governance» (Beunen, et al. 2015). Sin embargo, debido a la propia lógica de la sociedad con las nociones de autoorganización y autonomía, contrastan con el concepto de Luhmann de autorreferencialidad (y que incluye el «cierre operativo» ${ }^{2}$ ) que compone los sistemas sociales complejos. En base a ello, es posible llegar a la conclusión de que no es posible iniciar un direccionamiento de la sociedad del exterior, sino que debe iniciarse en su interior, por lo que resulta superfluo referirse a formas externas de gobernanza.

Por otro, el contexto externo de la gobernanza dos fenómenos recientes han impactado en su concepción: la aparición de la crisis económica y financiera mundial en el año 2007 y la libre competencia asociada, y el modelo de «autorregulación del mercado». Mientras que el primero, ha tenido impactos y secuelas sociales que siguen todavía vigentes, y que han conducido a que la gobernanza (y la teoría de la dirección ${ }^{3}$ ) se encuentre en una situación de escepticismo, crisis, o inmersa «en un montón de ruinas» (Willke, 2014). Ambos modelos han producido profundas asimetrías y huellas en todas las sociedades en las prácticas y modelos de gobernanza han sembrado decepción, de tal forma que se manifiesta una confusión y resignación generalizadas. Los modelos de gobernanza económica y planificación están totalmente desacreditados, al igual que también lo están los modelos que se basan exclusivamente en el mercado. Estas limitaciones se han puesto de manifiesto en múltiples ocasiones en las sociedades occidentales y las ilustramos con dos ejemplos recientes: el referéndum de Italia (2016) y un plebiscito secesionista en Brasil (2011). Una respuesta adecuada a las limitaciones teóricas de este tipo de descripción y de extensión de la gobernanza en las diversas áreas de conocimiento, solo puede provenir de un replanteamiento conceptual y operativo del término.

En esta situación aparece necesario un debate teórico en la teoría de la gobernanza (que diferenciamos de la teoría del estado $)^{4}$ con el objetivo de clarificar la complejidad del término y que implique repensar ideas de autoorganización y autorregulación desde una perspectiva que la diferencie de las propuestas clásicas de gobernanza política, basada solo en la descripción normativizada de la coordinación entre actores e instituciones.

La hipótesis sustantiva que planteamos en este trabajo puede resumirse como el concepto de gobernanza en su plano operativo ha de sostenerse en los cambios funcionales de la sociedad que se manifiestan

\footnotetext{
1 También se vincula en relación con las políticas públicas (SORENSEN; Triantafillou, 2009).

2 El concepto de «cierre operativo» (Operative geschlossenheit) es central en la descripción autorreferencial de Luhmann. También ha sido de los mas controvertidos.

3 La palabra inglesa governance se equiparó con governing (como forma procedimental de gobernar). En Alemania se ha utilizado indistintamente el término inglés governance utilizándose como sinónimo de Steuerungstheorie (teoría de la dirección). Thomas Gawron, Steuerungstheorie, Policy-Forschung und Governance-Ansatz: zum verfehlten Governance-Konzept der Regionalforschung, 2010.

4 Jörg KLAWITTER, Staatstheorie als Steuerungstheorie? 1992: Staatstheorie als Steuerungstheorie? Vorüberlegungen im Hinblick auf ein staatstheoretisch fundiertes, akteurzentriertes und verhandlungsorientiertes Netzwerk polyzentrischer Gesellschaften.
} 
en diversos planos, los cuales habitualmente han sido observados de forma limitada e imprecisa. Su observación debe configurarse a partir de:

- Primero, con la observación e inclusión de los cambios en los sistemas funcionales sociales.

- Segundo, que incluya una concepción diferenciadora que contemple la conexión entre territorio y política.

- Tercero, que en la lógica neosistémica se asuman los procesos de autoorganización de la sociedad.

La exposición que sigue a continuación sigue este orden, en primer lugar, exponemos los fundamentos de la gobernanza sistémica cuyas fuentes provienen de la teoría de sistemas autorreferenciales de Niklas Luhmann y las aportaciones posteriores de Helmut Willke, en particular analizamos la vinculación con el territorio y la política. En la parte segunda presentamos la idea de una gobernanza sistémica procesual que puede ser operativa para futuras observaciones de fenómenos políticos y que posibilite un vínculo conceptual y operativo con el territorio. Por último, a modo de ilustración, exponemos la relación entre los conceptos y variables con los casos de Italia y Brasil.

\section{FUNDAMENTOS TEÓRICO-CONCEPTUALES DE LA GOBERNANZA SISTÉMICA}

La crisis económico-financiera iniciada en el año 2007 ha contribuido a un «desprendimiento» de las capacidades de la gobernanza, al poner de manifiesto la limitada capacidad de gestión efectiva interna (endógena) para hacer frente a los desafíos exógenos, de tal forma que este tipo de fenómenos se han convertido en permanentes. Estudios destacan la «merma de performance directiva del gobierno» (Poblete, 2014:27), cuyos síntomas de «ingobernabilidad» en las sociedades occidentales ya fueron visibles en las últimas décadas del siglo pasado (Luhmann, 1981) ${ }^{5}$.

En la perspectiva teórica sistémica, las sociedades se presenta de forma heterárquica fundada a partir de las semánticas directrices de los diversos sistemas sociales («sin centro» y «sin vértice»). La sociedad no se concibe más en términos jerárquicos (clase sociales) sino como "policéntrica» (Willke, 1997; 1996; 1992) o "funcionalmente policéntrica», o sea, un funcionamientos de los diversos sistemas (educativo, político, jurídico) en que sus funciones son ejercidas en forma de red, con «direccionamiento descentralizado» (Glagow; Willke, 1987) y en la que cada sistema funciona a según su propia lógica.

\subsection{Política y territorio}

El sistema político contemporáneo está sujeto y condicionado también por una serie de mecanismos ineficaces que interfieren en su éxito o fracaso, como son la burocracia parlamentaria o la incapacidad de los partidos de asumir los cambios estructurales internos necesarios. En la concepción de sistema autorreferencial, la política posee la capacidad de dirección (o de gobernanza) con la creación de sus propias operaciones y estructuras, y de las formas en que observa su entorno, los códigos utilizados o la función del sistema político (Gonnet, 2010; Hellmann; Fischer; Bluhm, 2003; Holz, 2003).

Fenómenos políticos recientes han mostrado la limitación de la capacidad de gobernanza por parte del gobierno y su conexión con el territorio. Estas limitaciones en las funciones ponen en duda su facultad y capacidad para actuar y desempeñarse como agente director de la sociedad, al colocar en las democracias occidentales la disyuntiva en un plano extremo de suma cero. Cuanto mayor la determinación política gubernamental, «tanto menor» la determinación de otro tipo (en forma de autoorganización social), o en una palabras más politizadas, «cuanto más Estado, tanto menos libertad» (Luhmann, 1993:51) ${ }^{6}$. Su controvertida sentencia ya que el Welfare state viene a significar más intervención en las políticas públicas y en la presencia institucional (ambas necesarias como veremos, de la región de la Amazonia). Su propuesta es clara en este sentido, dirigiendo su análisis a los procesos de diferenciación social de las sociedades actuales.

La autoorganización y la autorreferencia aparecen como ideas clave en la nueva forma de organización social en el contexto de cuestionamiento del sistema político contemporáneo y de sus semánticas y prácticas

\footnotetext{
5 En la perspectiva de la diferenciación política destaca que la gobernanza se ha relacionado con tres dimensiones de la actividad política. Parinós (2008) distingue entre: 1) con las formas de organización territorial y de la administración del estado (polity); 2) con la forma en que son repartidas las responsabilidades y con la tomada de decisiones (politics); 3 ) con la sociedad civil (en especial las ONGs) con el enfoque de la sociología política.

6 Para Luhmann (1993: 55): «la autorreferencia política es, en consecuencia, el producto de la diferenciación social específica de los sistemas políticos».
} 
benefactoras traducidas en el Estado de bienestar y sus posibilidades de inclusión social de los sectores desprotegidos o excluidos.

Surge el interrogante si podemos referirnos a la existencia de sistemas autoorganizados en las sociedades actuales En un plano teórico autopoietico (o sea referente a los seres vivos) o con referencias antropológicas, los seres humanos en su dimensión biológica y psicológica son sistemas autoorganizados. La transposición de esta idea al plano social es problemática. Al producirse la autoorganización de un sistema social, este desarrolla también un ciclo de vida o desarrollo propio (Leydesforff, 2015:14-28; Valera; Maturana 1984). La dificultad aparece a la hora de extender esta cualidad a los sistemas sociales al disponer y desarrollar otros conceptos operativos. Un sistema también se autorregula y aprende de los errores del pasado. La autorreflexividad interna aparece como una condición para su autoorganización. No obstante la autoorganización en un sistema social no puede considerarse como una característica del sistema, puesto que no poseen todos los sistemas esta capacidad, por lo que es necesario «mantener» esta premisa como hipótesis (Leydersdorff, 2015:22).

La politóloga Renate Mayntz amplia la idea sobre la capacidad de autorregulación de la sociedad civil: "Governance se refiere a la totalidad de las formas existentes de reglamentación colectiva de los hechos sociales: desde la institucionalización de la autorregulación de las sociedades civiles, las diferentes formas de cooperación de los actores estatales y privados hasta la acción soberana de los atores estatales» (Mayntz, 2004:66). La definición hace referencia a la acción recíproca entre el sistema político, el sistema de los destinatarios con su entorno social. El direccionamiento estatal (o gobernanza política) se diferencia de las formas no-estatales de dirección de la sociedad (autonomía de los respectivos sistemas) y de las formas en que la orientación-finalidad-medio se oponen a las instancias estatales y a los órganos de toma de decisiones gubernamentales (cuyos resultados en el ámbito político han la aceleración del neoliberalismo con las prácticas de la desestalización y privatización).

\subsection{Direccionamiento estatal y gobernanza}

Des de la década de los años ochenta los debates técnico-sociales y en la ciencia política se indican los problemas estructurales en que se enfrentaba el sistema político en las sociedades occidentales (Offe, Habermas, Luhmann). Entretanto, la política ha asumido cada vez más con mayor dificultad la «dirección de la sociedad» (o de gobernanza), llegando en la actualidad a una situación límite de su capacidad de dirección. Bajo el término de dirección de la sociedad, el Estado (institutiones y mediante parlamentos, gobiernos y administraciones) que actúa como el único centro de control político. En un primer momento, las publicaciones focalizaron su papel cambiante en la toma de decisiones en el plano gubernamental sobre las respectivas instancias y subesferas socialmente controladas (como son los grupos de interés o los sindicatos) y en las organizaciones políticas representativas. En la perspectiva sistémica, los sistemas parciales se ocupan de la correspondiente función parcial sustantiva, que en el caso de la economía es la confección de bienes para la satisfacción, en la ciencia para la procura de verdades, en el sistema de salud para el mantenimiento biológico de los miembros de la comunidad, etc.

\subsection{Diferenciación funcional de la sociedad y operaciones}

La perspectiva funcional de análisis de la sociedad significa la compresión de un proceso mediante el cual los sistemas parciales que la componen (como el educativo, jurídico, económico) se distinguen y diferencian entre ellos, mediante asumir operaciones y funciones específicas del entorno y de otros sistemas y subsistemas. En cada sistema es esencial la información producida y emitida.

El marco en el que tienen lugar estas operaciones procede de la descripción de la sociedad caracterizada por la ausencia de un «centro» y en la que los sistemas (subsistemas) operan independientemente unos con otros. La especialización funcional de cada sistema no se basa en una multifuncionalidad difusa, sino que permite a cada subsistema, un tipo específico de sensibilidad de las formas de abordar los fenómenos. Esta forma de operar y la sensibilidad de cada sistema, se manifiestan al adaptarse al entorno, o sea, a las formas de operar de cada fenómeno. Los códigos binarios en que opera el sistema sirven para la reducción de la complejidad a la vez que muestran su grado de especialidad (en el caso del sistema jurídico el código binario es legal/ilegal). Sin embargo, dada la complejidad de las sociedades actuales, estos códigos binarios pueden resultar insuficientes. 
Simultáneamente, los sistemas deben adaptarse a las exigencias de otros sistemas, aunque la adaptación no es suficiente para conseguir la necesaria integración social. Cada sistema parcial debe proporcionar siempre más información detallada sobre sus respectivas operaciones procedentes de la política (sistema político), con lo que deben traducir el su lenguaje (en forma de tomada de decisiones vinculantes, decretos, leyes). Con el aumento de la diferenciación funcional de las sociedades actuales, también se sofistican las posibilidades de dirección, de tal forma que se vuelve prácticamente imposible su direccionamiento y también significa que la política debe estar en el nivel más elevado de las informaciones. Ello repercutirá de nuevo en el aumento de la diferenciación funcional y una mayor necesidad de direccionamiento.

Como ejemplo, el sistema debe ofrecer información sobre la política educativa o los cambios en el sistema de educación de un país, o la prioridad de función del sistema político administrativo será ofrecer unas directrices de acción a la sociedad mediante políticas públicas u otro tipo de acciones. En la perspectiva sistémica la prioridad funcional de un sistema se dirige a su entorno y no al sistema en su conjunto.

\subsubsection{Diferenciación funcional y «triple diferenciación»}

La conexión de causa-efecto en la diferenciación funcional de las sociedades complejas actuales se asocia a las formas de gobernar con los cambios estructurales que están ocurriendo en ellas, o sea, los cambios en la diferenciación funcional de la sociedad y en la acción de gobernar (Aguilar, 2007; Poblete, 2014). La existencia y disposición de una variedad de instrumentos de la gobernanza es uno de los determinantes más importantes para la estabilidad social y la continuidad del desarrollo y evolución. A diferencia de los otros sistemas parciales, la política constituye una «meta-contribución» para la regulación social (Schimank; Glagow, 1984:10) y como «factor de cohesión» en tanto que garantiza la integración de los otros sistemas parciales.

Resulta evidente que esta descripción de la sociedad tiene implicaciones para el sistema político. A diferencia de los sistemas políticos tradicionales caracterizados por la diferenciación bidimensional (dominados y dominantes) con los actuales sistemas políticos se fundamentan en la diferenciación subsistémica de la «triple diferenciación» entre Política, Administración y Público (Luhmann, 2007:62-63) ${ }^{7}$. Cado uno de estos subsistemas que la componen pasa a ser como un sistema autónomo en sus operaciones especiales, simultáneamente puede ser percibido como el entorno de otros subsistemas. Así pues el entorno del sistema económico puede ser el sistema de salud o el entorno del sistema jurídico puede ser el sistema económico.

\section{GOBERNANZA SISTÉMICA: PREMISAS TEÓRICO-CONCEPTUALES CONFIGURADORAS}

La idea de gobernanza que utilizamos aquí proveniente de los conceptos teóricos de la teoría de sistemas autorreferenciales y el «constructivismo operativo» de Niklas Luhmann ${ }^{8}$, que se amplía con las recientes aportaciones e interpretaciones post-sistémicas procedentes de la sociología comunicacional (Leydesdorff, 2015) y de las corrientes interaccionistas que contemplan la inclusión de un sistema de conciencia sujeto a comunicaciones lingüísticas.

Dos ideas contrales componen el debate de la gobernanza que presentamos. La primera, asume la teoría de sistemas autorreferenciales como referencia; la segunda profundiza los problemas relativos a la diferenciación funcional de la sociedad. Ambas son necesarias para el análisis de la gobernanza y tienen sus orígenes en las últimas décadas.

Para el ámbito comunicacional recurrimos a las aportaciones constructivistas y la noción de autopoiesis procedentes de los neuropsicólogos Humberto Maturana y Francisco Valera (1984), poco conocidas en la ciencia política y de la administración pública. El concepto de autopoiesis en su planteamiento original -para Maturana y Valera: «dominios lingüísticos de la conciencia humana» (Maturana y Valera, 1984:137). El concepto nos posibilita retomar la noción de autoorganización (y la autoproducción) de los sistemas en una perspectiva de sistema vivos, y en concreto de construcción de sus propias identidades mediante un sentido

El sociólogo esloveno Slavoj Žižek, aunque no se fundamenta en estos postulados sistémicos luhmannianos, se remite a Peter Sloterdijk, al reconcer que: «hoy en día la lucha de clases ya no es típicamente marxista», con el argumento que el nuevo choque es «entre los que están dentro y los que quedaron fuera» (2016). Slavoj ŽlŽEK "Per una diversa lotta di classe", en: http://sollevazione. blogspot.com.br/2016/05/per-una-diversa-lotta-di-classe-di.html.

${ }^{8}$ No es sencillo caracterizar el constructivismo que Luhmann denomina «constructivismo operativo». En su obra hace referencia a dos corrientes del construccionismo epistemológico: el construccionismo de inspiración piagetiana -denominado por Luhmann como «genética-constructivista»- y el constructivismo radical de von Glasersfeld. 
creado por un ser humano. El concepto permite asumir en modo operativo el nivel subjetivo de la acción individual no vinculada a los actores colectivos.

La capacidad de pensamiento está sustentada por dos ideas centrales la forma de concebir la estructura y la semántica de proceso. Con ellas nos diferenciamos del individualismo metodológico que prioriza la estructura y los cambios por medio de elementos individuales de los individuos. Por otro lado, la idea de proceso que utilizamos procede de las ideas del interaccionismo simbólico. Se postula como premisa básica que la sociedad está formada por individuos, y que por lo tanto son sujetos con una composición psíquica y con capacidad de pensamiento. La capacidad de pensamiento reside en la mente, concebida no como una estructura sino como un proceso ininterrumpido de cada ser humano.

Las estructuras -ya sean medios simbólicamente diferenciados o en forma de diferenciación-, son comunicaciones en constante transformación. Son comunicaciones dinámicas que al ser utilizadas se transforman. La similitud entre las premisas interaccionistas y las operaciones comunicacionales en la teoría luhmanniana, aparece con el reconocimiento central del proceso de interacción social que configura la capacidad de pensamiento. Esta capacidad es configurada por la socialización y por la interacción social y su forma específica en la socialización (Berger; Luckmann [1968]2003). Se distancia de la concepción estructural sistémica ya que el pensamiento no lo consideramos como una estructura sino como parte de la mente que se diferencia del cerebro fisiológico.

La adopción de la idea de concebir la capacidad de pensamiento como un proceso en un sentido interaccionista tiene consecuencias para el abordaje de la idea de gobernanza sistémica. Nos permite operar en el plano sistémico en el nivel subjetivo del sistema. No profundizaremos en esta línea aquí, indicando tan solo las posibilidades operativas de introducir este nivel observacional. En la propuesta, asumimos como presupuesto (o hipótesis secundaria) la política ejerce un tipo de direccionamiento o de «meta-contribución» a la totalidad de la reproducción social, en cuanto que garantiza una integración con los restantes subsistemas. Luhmann le atribuye una posición especial al definirla como «el destinatario último» y «última instancia» además de un sentido (Luhmann, 1993:156) ${ }^{9}$.

\subsection{Sistema e integración}

Como sistema nos remitimos a la premisa definitoria de Luhmann (1984) según la cual un sistema se convierte en sistema: «si por medio de sus propias operaciones el sistema se vuelve a si mismo sistema». La autorreferencialidad en sus operaciones lo caracterizan como un sistema cerrado cuya principal diferenciación consiste en la diferencia con el entorno. Los tres sistemas principales en que se sustenta la sociedad (jurídico, económico y político-administrativo) poseen cada uno de estos un entorno interno y otro externo. En el sistema político el entorno interno está formado por otros partidos, parlamento, electores, familia, mientras que el externo se configura por los otros sistemas y subsistemas y sus entornos.

Como integración nos referimos a la contemplación de forma no jerarquizada los diferentes niveles de observación (meso, macro, micro) y de asumir además el nivel subjetivo. Con el nivel subjetivo retomamos la inclusión de sujeto en el sentido antropológico y representado por el interaccionismo simbólico, definido como un «sujeto con capacidad de pensamiento» (según los postulados Berger y Luckmann, 2003) ${ }^{10}$. No solo retomamos los imperativos del sistema general de la acción (resumidos en sistema cultural, sistema social, organismo conductual y sistema de la personalidad parsoniano) sino asumir los principios de ser humano esbozados por el interaccionismo simbólico.

La integración es posible operativizarla en los niveles observacionales. Posee la capacidad operativa de filtrar e integrar comunicaciones procedentes de los subsistemas funcionales y de transmitirlas nuevamente de forma vinculante. El principio de integración no significa una «meta gobernanza», sino que la idea que proponemos se limita a la hipótesis de configuración teórica de una operación observacional.

En un plano operativo tiene la posibilidad de observar los códigos de los subsistemas sociales con el objetivo de obtener informaciones para analizar los cambios funcionales y emergentes de cada uno de estos (lo que induce a una cierta semejanza con el método heurístico de conocimiento). La operación tiene la ventaja de obtener una visión general intersistemas mediante una serie de informaciones (cualitativas y cuantitativas) para la posterior toma de decisiones.

\footnotetext{
9 Willke dibuja la posibilidad de que un sistema pueda asumir la función de orientar contextualmente a otros sistemas o actores colectivos.

10 La capacidad de pensamiento del ser humano en el sentido sociológico está ampliamente desarrollada por Blumer (1969), Manis y Meltzer (1978) y Mead (1975).
} 
Opera exclusivamente por medio de comunicaciones que son simplificadas en base al conjunto de las diferenciaciones funcionales de los sistemas de la sociedad y los códigos que emanan de cada sistema. La inferencia de los códigos corresponde al observador para sobrepasar la posibilidad buen/mala gobernanza para ampliarse en extensiones de la gobernanza buena (con deficiencias, rectificable en algunos aspectos, o mala) (Figura 1).

\section{FIGURA 1. EXPOSICIÓN ESQUEMÁTICA DE LOS SISTEMAS PRINCIPALES Y OPERACIONES QUE INTEGRAN LA IDEA GOBERNANZA SISTÉMICA}

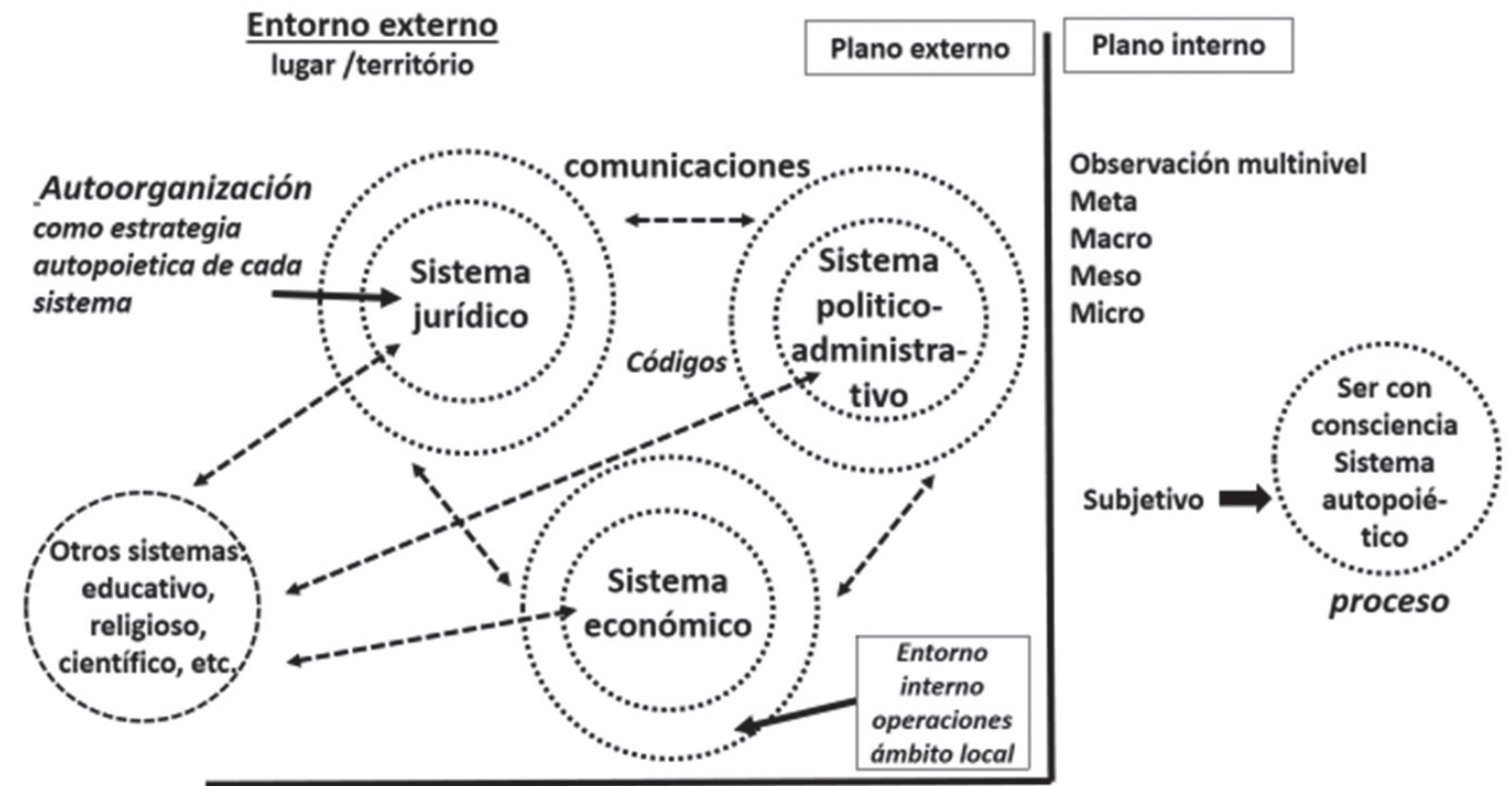

Estructura, dinamismo : capacidad de transformación del sistema social en su totalidad

Fuente: autor

En la representación esquemática que proponemos, diferenciamos entre un plano externo y un plano interno. En el plano externo, como perspectiva de la observación, se presentan los tres principales sistemas (según Luhmann, económico, político-administrativo y jurídico) y las comunicaciones posibles con otros subsistemas (científico, religioso, etc.). En territorio aparece como externo de cada sistema, y está compuesto por la sociedad civil (o Público, en Luhmann) y las correspondientes comunicaciones en forma de acciones procedentes de esta. En plano interno comprende la perspectiva propia del observador en su operación de autoobservarse. Consideramos que los niveles meta, meso y micro son insuficientes y no ofrecen la posibilidad de una observación más precisa. Por ello, proponemos el nivel subjetivo, es decir, a los individuos no organizados que participan esporádicamente y eventualmente en diversas acciones públicas (consultas, talleres, espacios deliberativos, etc.)

\section{GOBERNANZA POLÍTICA Y TERRITORIO: ITALIA Y BRASIL}

Nuestra pretensión no es exponer una inestigación comparada exhaustiva entre países, sino que el objetivo es ilustrar los conceptos expuestos mediante de desripción de la interacción en el sistema político y en el administrativo con el territorio en dos administraciones y sistemas políticos diferentes. Ambos países han experimentado dos fenómenos recientes que de alguna forma u otra ponen de manifiesto la idea de la gobernanza política y su conexión con el territorio (o gobernanza territorial): el referéndum constitucional en Italia (2016) y un plebiscito territorial en una Unidad Federativa (estado) en Brasil (2011).

En las diversas vertientes operativas de la gobernanza política, la administración y el territorio aparecen estrechamente relacionadas. En la perspectiva sistémica, el territorio es el espacio y el espacio o lugar donde están inscritos los votantes, el lugar en donde los ciudadanos expresan y reciben los servicios públicos y donde se aplican las políticas públicas. Para la administración el territorio es el lugar en que están administradas las demandas de los ciudadanos y el lugar de deliberación. Los dos fenómenos de acción política son diferentes, 
aunque también guardan ciertas similitudes. El plebiscito es una consulta a la ciudadanía antes de que sea constituida una ley (se remite a aprobar o rechazar la propuesta de ley), mientras que el referéndum es una consulta a la ciudadanía después de que una ley ha sido aprobada por un parlamento, es decir, se sanciona (referenda) una propuesta operada por el Estado. Salvando estas distancias en cuanto a intencionalidad de cada caso en la perspectiva de la gobernanza política permite observar algunas similitudes en el plano observacional.

Italia y Brasil son dos países con diferencias de el desarrollo funcional. Salvándo esa distancia, en el plano ilustrativo de análisis sistémico que nos proponemos, poseen algunos elementos que nos permiten una comparación aproximada. En primer lugar, se trata de dos regímenes políticos republicanos y federativos: Italia es definida como republica parlamentaria con un sistema federalista ${ }^{11}$ y Brasil, es constituido como una república federativa estructurada bajo la forma de un presidencialismo de coalición ${ }^{12}$.

La constitución brasilera aprobada en 1988 fue aprobada para dejar atrás de forma consensual entre las fuerzas políticas la dictadura militar y pensada con una clara separación de poderes en un sistema presidencialista.

Ambos países están inmersos en una fragmentación del sistema político y administrativo, y en lo que se califica de "systemic corruption» y "organised corruption» (Vannucci, 2009) (extensible a otras democracias occidentales de países mediterráneos). Como característica diferenciadora para los objetivos de este escrito, es la posición inicial oficial del sus respectivos gobiernos respecto a las consultas realizadas. La descripción la realizamos a partir de tres variables explicativas: 1) la perspectiva política y la extensión con el territorio; 2) el direccionamiento estatal y la gobernanza; 3) la diferenciación funcional de la sociedad.

\subsection{El referéndum constitucional en Italia}

\subsubsection{Antecedentes}

En Italia la consulta partió de un proyecto gubernamental para todo el país. El sistema político administrativo italiano posee unas peculiaridades históricas y sociales que lo diferencian de otros sistemas democráticos occidentales ${ }^{13}$. Entre estas destacan unos fenómenos estructurales que han lastrado su desarrollo, no exclusivos de Italia (que pueden extenderse a otros países mediterráneos) ${ }^{14}$ :

- Partitocrazia, en la que los partidos políticos controlan la provisión y ocupación de los puestos públicos, anulándose la profesionalidad y la meritocracia. El amiguismo impera en la administración pública mediante el concepto de «restauración» ${ }^{15}$.

- Lotizzazione, vinculada con el reparto de las cuotas de puestos públicos entre los partidos en el poder o de la coalición gubernamental.

- Tangentopoli, («la polis de los sobornos») corrupción de los políticos y los funcionarios.

Aunque esto fenómenos con otras denominaciones aparecen en prácticamente todas las democracias occidentales en Italia han adquirido un elevado grado de simultaneidad en los diferentes niveles de gobierno.

A estos fenómenos aparecen otros no menos importantes como es la «patrimonialización», que se manifiesta en las formas de selección de los funcionarios públicos, los cuales en un porcentual elevado proceden de las zonas rurales meridionales del país. En estas zonas es común que el trabajo en la administración sea un trabajo complementario por la tarde o incluso por la mañana durante jornada laboral ${ }^{16}$.

11 Ver: Gianfranco PASQUINO, La Costituzione in trenta lezioni. Utet, 2015.

12 Ver: Octávio AMORIM NETO, (2006). Presidencialismo e Governabilidade nas Américas. Rio de Janeiro. José Antônio CHEIBUB; Adam PRZEWORSKI; Sebastian SAIEGH, (2002). "Governos de Coalizão nas Democracias Presidencialistas e Parlamentaristas", Dados, núm. 45.

13 La Constitución italiana de 1948 y pensada originalmente para impedir cualquier tipo de régimen autoritario mediante un complejo sistema de contrapoderes denominado como «bicameralismo imperfetto» (Pasquino, 2015).

14 Seleccionadas por el Istituto di Reserce Sulla Pubblica Administrazione, 2014.

15 Las formas de adjudicación de los puestos de dirección son un campo de observación para entender cómo se ha reubicado el equilibrio entre la política y la administración. Se trata de una «reconstrucción» permeada por un proceso tácitamente de «restauración». Este puede ocurrir al vaciar el principio de separación entre la política y administración que es la condición necesaria para distinguir las funciones, las tareas y responsabilidades. Stefano BATTINI e Luigi FIORENTINO, "Venti anni di 'politica e amministrazione in Italia', Istituto di Ricerche sulla Pubblica Amministrazione”. IRPA Working Paper, Policy Papers Series, núm. 1/2014, pág. 74.

${ }_{16}$ La mitad de los empleados públicos han entrado en la función pública sin pruebas de ingreso con contratos temporales, como interinos o funcionarizándose mediante leyes y decretos especiales. La administración pública es concebida como una alternativa que 
El reciente referéndum en Italia (4 de diciembre de 2016) sobre la propuesta de cambios en la Constitución para acabar con el llamado bicameralismo (que otorga la misma fuerza al Congreso y al Senado) orientados reorganizar el aparato administrativo y territorial del Estado ha puesto al descubierto una serie de problemas ${ }^{17}$. Las razones dadas por los partidarios de la reforma son multiples, diferenciándose entre los argumentos sobre los motivos del referéndum ${ }^{18} \mathrm{y}$ las opciones, entre las que destacan:

- Transformación de la bicameralismo en «bicameralismo diferenciado».

- Introducción de un proceso legislativo más ágil a partir de un nuevo procedimiento ordinario que no precisa ser aprobado necesariamente las dos cámaras.

- Ahorro estimados en varios cientos de millones de euros, derivado de la supresión del Consejo Nacional de Economía y Trabajo de Italia $(\mathrm{CNEL})^{19}$ al reducir el número de senadores, el límite de la compensación entregado a los concejales y la prohibición de financiación grupos políticos regionales.

- Superación de muchos de los conflictos de autoridad entre el Estado y las regiones en el ejercicio del poder legislativo por medio de una reducción de la autonomía regional. Medida se justifica por los escándalos y la mala gestión de los recursos públicos en diferentes gobiernos locales.

Aparentemente la propuesta ha tratado de introducir las pautas para una modernización del aparato político administrativo italiano con el objetivo de ahorrar costos de duplicidades y hacerlo más eficiente. La iniciativa del gobierno ya aprobada previamente por el Parlamento italiano y propugnada por el entonces Primer ministro Renzi ${ }^{20}$, ha puesto de manifiesto profundas divisiones de concepción que sobrepasan la diferenciación bidimensional y el binomio izquierda-derecha ${ }^{21}$ y el contraste Norte (desarrollado) - Sur (agrario) del país (Cuadro 1).

CUADRO 1. POSICIÓN DE LOS PARTIDOS POLÍTICOS ANTE LA PROPUESTA GUBERNAMENTAL

\begin{tabular}{cll}
\hline Opción & Partido & Ideología* \\
\hline $\mathrm{Si}$ & Partido Democrático (PD) & $\begin{array}{l}\text { Socialdemócrata. Primer ministro: Matteo Renzi 2015; } \\
\text { desde 2016 Paolo Gentilone }\end{array}$ \\
\hline $\mathrm{Si}$ & Nueva Centroderecha (NCD) & Derecha conservadora \\
\hline $\mathrm{Si}$ & Alianza Liberal Popular (AL-A) & Centro \\
\hline $\mathrm{Si}$ & Elección Cívica (SC) & Liberal \\
\hline $\mathrm{Si}$ & Partido Socialista Italiano (PSI) & Socialdemócrata \\
\hline $\mathrm{Si}$ & Actúa ! (F!) & Federalista \\
\hline $\mathrm{Si}$ & Centro Democrático (CD) & Izquierda cristiana \\
\hline $\mathrm{Si}$ & Italia de los Valores (IdV) & Populista \\
\hline $\mathrm{Si}$ & Radicales Italianos (RI) & Liberalismo \\
\hline $\mathrm{No}$ & Movimiento5 Estrellas (M5E) & Populismo conservador \\
\hline No & Forza Italia (FI) & Liberal conservador, derecha \\
\hline
\end{tabular}

ofrece estabilidad y carrera. La selección de los funcionarios se produce por medio de la lotizzazione y la partitocrazzia, reduciéndose así la meritocracia y la competitividad. La administración pública es tan sólo un medio para lograr la estabilidad.

17 Los proyectos e intentos de reforma constitucional y de presidencialización en la Segunda República Italiana ya se vienen gestando desde hace más de un cuarto de siglo (Catelani, 2016; Ruiz, 1992; Abagnale, 1990).

18 El profesor Raniero LA VALLE en "Italia: la verdad sobre el referendum de Renzi", Sin Permiso.

19 Ver: http://www.cnel.it/home.

20 Giovanna CASADIO, Giovanni CAGLIARDI, "Referendum costituzionale, minoranza Pd non firma. Renzi: "C'è una parte che si oppone a tutto", La Repubblica, 20 aprile, 2016.

21 Las fuerzas políticas que apoyaron al gobierno en el parlamento y que votaron a favor de la reforma (Partido Demócrata), los grupos de interés de las regiones formados por miembros en el nuevo centro-derecho y UDC, ALA (compuesta en gran parte de la antigua inscrito en Forza Italia) y otros partidos más pequeños, como el Centro Democrático, el Partido Socialista italiano y Elección Cívica. Entre los que se opusieron a los cambios constitucionales además del Movimento 5 Stelle, Italiano Izquierda-Izquierda, Ecología y Libertad, Liga Norte y Hermanos de Italia, también Forza Italia, que en un primer momento de reforma incurrió la propuesta. 
GAPP. Nueva Época - N.1, noviembre 2017 - ISSN: 1989-8991 - DOI: 10.24965/gapp.v0i18.10383 - [Págs. 62-80]

Referéndum constitucional en Italia y plebiscito territorial en Brasil. Un enfoque de sistémico de gobernanza Josep Pont Vidal

\begin{tabular}{lll}
\hline No & Izquierda Italiana $(\mathrm{SI})$ & Socialismo democrático \\
\hline No & Liga Norte $(\mathrm{LN})$ & Regionalista, derecha conservadora \\
\hline No & Hermanos de Italia $(\mathrm{Fdl})$ & Conservador regionalista, euroescéptico \\
\hline No & Unión de Centro (UDC) & Democracia cristiana \\
\hline No & Conservadores y Progresistas (CR) & Conservador \\
\hline No & Posible $(\mathrm{P})$ & Progresista \\
\hline No & Federación de los Verdes (FdV) & Política Verde ecologista \\
\hline No & Partido Refundación Comunista (PRC) & Marxismo radical \\
\hline No & Partido Comunista Italiano (PCI) & Marxista \\
\hline No & CasaPount Italia (CPI) & Neofascista \\
\hline No & Fuerza Nueva (FN) & Neofascista, ultranacionalista \\
\hline
\end{tabular}

* La clasificación ideológica de un partido es en parte objetiva y subjetiva. Puede ser que un partido no se defina en sus estatutos como extremista, pero sus acciones y práctica pueden indicar un radicalismo.

Fuente: Italia, Ministerio del Interno, Informaciones según Camera - votazione núm. 1, seduta núm. 606 del 12-04-2016, en openparlamento, Openpolis 2016

\subsubsection{Resultados}

A pesar de tratarse de un referéndum para refrendar una propuesta de direccionar tomada «desde arriba» de la coalición de partidos y su campaña para el «si», el resultado fue un amplio triunfo del voto «no» (59,95\% de votos, frente al «sí» con 40,05 \%, con un índice de participación de 68,2 \%) y una abstención de casi 15 millones de italianos. El elevado grado de abstención que no significa desinterés por la política (apolítico) o voto de apoyo a la opción ganadora, sino también desinterés por la gobernanza política y la política tradicional o por los canales de participación (Cuadro 2).

CUADRO 2. VOTOS EN EL REFERÉNDUM (VOTOS) ${ }^{22}$

\begin{tabular}{lrr}
\hline Si & 12.709 .536 & $40,05 \%$ \\
\hline No & 19.025 .254 & $59,95 \%$ \\
\hline Válidos & 31.734 .790 & $99,19 \%$ \\
\hline No válidos y nulos & 263.126 & $00,81 \%$ \\
\hline Participación & 31.997 .916 & $68,48 \%$ \\
\hline Votantes registrados & 46.720 .943 & $100,00 \%$ \\
\hline Abstención & 14.723 .027 & \\
\hline
\end{tabular}

Fuente: Italia, Ministerio del Interno 2016

Los diagnósticos y las encuestas y sondeos de opinión previas encargadas por el gobierno o bien no fueron bien interpretados, o fueron ignoradas por las instancias políticas. Tampoco contemplan con suficiente profundidad, o indican también el voto «volátil» de última hora, aunque ya preveían una victoria por un margen mínimo.

Entre los efectos políticos el mas llamativo ha sido la renuncia del Primer ministro como jefe de gobierno al vincular el resultado del referéndum a su continuidad. Para el Instituto de Relaciones Internacionales ${ }^{23}$ el referéndum constitucional ha implicado un cambio profundo también en las relaciones de poder político:

22 En el referéndum han también participado los electores con residencia en el exterior (31 de diciembre de 2015) sumando un total de casi cinco millones. El porcentaje en el padrón total de los italianos residentes en el extranjero supera el 8 por ciento.

${ }_{23}$ Universidad Nacional de La Plata, Instituto de Relaciones Internacionales, http://www.iri.edu.ar/wp-content/uploads/2016/12/ referendum-constitucional-italia-francesca-staiano.pdf. 
GAPP. Nueva Época - N. 18, noviembre 2017 - ISSN: 1989-8991 - DOI: 10.24965/gapp.v0i18.10383 - [Págs. 62-80]

Referéndum constitucional en Italia y plebiscito territorial en Brasil. Un enfoque de sistémico de gobernanza

Josep Pont Vidal

«Este voto representa que el establishment ya no está más respetado por el pueblo, que vive cada vez más en un desconcierto debido a la enorme e insostenible distancia que lo aleja de sus representantes».

\subsubsection{Análisis sistémico}

Para los objetivos del artículo nos limitaremos al análisis sistémico-funcional. En la perspectiva sistémica presentamos tres hipótesis que pueden sintetizarse en:

- Primera. La política como «meta contribución» entendida como factor para la reproducción social, o como «factor de cohesión» se ha mostrado limitada. Las posibilidades de autoorganización de la sociedad (de los sistemas) se han manifestado «hacia fuera», al sobrepasar los intereses concretos del partido gubernamental (para el caso de los sindicatos: Regalia, 2009; Cerri; Soli, 2009; Instituo di Recherche Economiche e Sociale de Veneto, 2008) ${ }^{24}$. Los códigos emanados por el sistema politico han sido recibidos por los otros subistemas de forma diferente o insuficiente (Cuadro 3 ).

CUADRO 3. SISTEMAS PRINCIPALES, OPERACIONES Y CAMBIOS FUNCIONALES

\begin{tabular}{lll}
\hline & Cambios funcionales y operaciones cuantificables & Abertura operativa \\
\hline Sistema económico & Apertura a la globalización. & Elevada \\
& $\begin{array}{l}\text { Tasa de informalidad laboral: } 9.8 \% \text {, entre las mujeres 11.0*. } \\
\text { Existencia de la Mafia en Mezzogiorno y Sicilia }\end{array}$ & Nula \\
\hline Sistema educativo & Censo 2011. Tasa de alfabetización $99 \%$ & Alto \\
\hline Sistema científico & Cooperación público-privada (universidades-empresas) & Elevada \\
\hline Sistema jurídico & Gobierno abierto y Justicia abierta & Alta \\
\hline Sistema religioso & $15 \%$ no religiosos, $8 \%$ ateos, sin religión $5.8 \%{ }^{* *}$ & Media \\
\hline
\end{tabular}

* https://www.istat.it/it/files/2015/03/IWP_1_2015.pdf.

** Global Index of religion and Atheism, 2013.

Fuente: autor

- Segunda. Las dos opciones posibles han sido percibidas como insignificantes en cambios de la vida de las personas, otras propuestas alternativas de reforma constitucional no han sido contempladas en el referéndum, o el principio de no participar en los canales tradicionales con propuestas de otro tipo de política no institucional. La abstención, como los resultados adversos a la propuesta gubernamental ponen en el punto de mira el concepto de gobernanza. ¿Se basó en la observación previa en los cambios funcionales de los diversos sistemas parciales de la sociedad italiana?, ¿qué esquemas conceptuales han sido aplicados?. Genera dudas sobre el tipo de diferenciación funcional aplicada. Aparece necesario establecer la conexión sistémica dinámica de causa contextual-efecto en la diferenciación funcional que asocie las formas de gobernar con los cambios funcionales estructurales (económicos, sociales, culturales) que han tenido en las regiones de Italia.

- Tercera. Las regiones con mayor índice de respuesta negativa al referéndum fueron las regiones industriales del norte y las poco industrializadas y agrarias del sur ${ }^{25}$. La fractura política y social profunda, entre regiones va también más allá de las opciones políticas (y del eje clásico izquierdaderecha). Sobrepasa los parámetros clásicos y plantea profundos problemas y concepciones a la gobernanza política y a la gobernanza en general. Ha puesto de manifiesto una fractura política y social profunda, entre regiones y que va también más allá de las opciones políticas (y del eje clásico izquierda-derecha) al sobrepasar los parámetros clásicos y plantear profundos problemas y concepciones a la gobernanza política y a la gobernanza en general (diferenciación tridimensional).

24 En el ámbito asociativo los sindicatosson un interesante indicador. El 33\% de tasa de afiliación está repartido entre los principales sindicatos confederales (15\% CGIL, 12\% CISL, 6\% UIL). CGIL. Casi la mitad de las afiliaciones (49\%) proviene de trabajadores que no están en activo (estudiantes, desempleados y jubilados).

25 No menos importante aqui es la existencia de capital social en el territorio, en el que la mafia constituye una variable. 
FIGURA 2. MAPA CON LA VICTORIA DEL SI O DEL NO, SEGÚN LAS REGIONES, PROVINCIAS, COMUNAS Y MUNICIPIOS

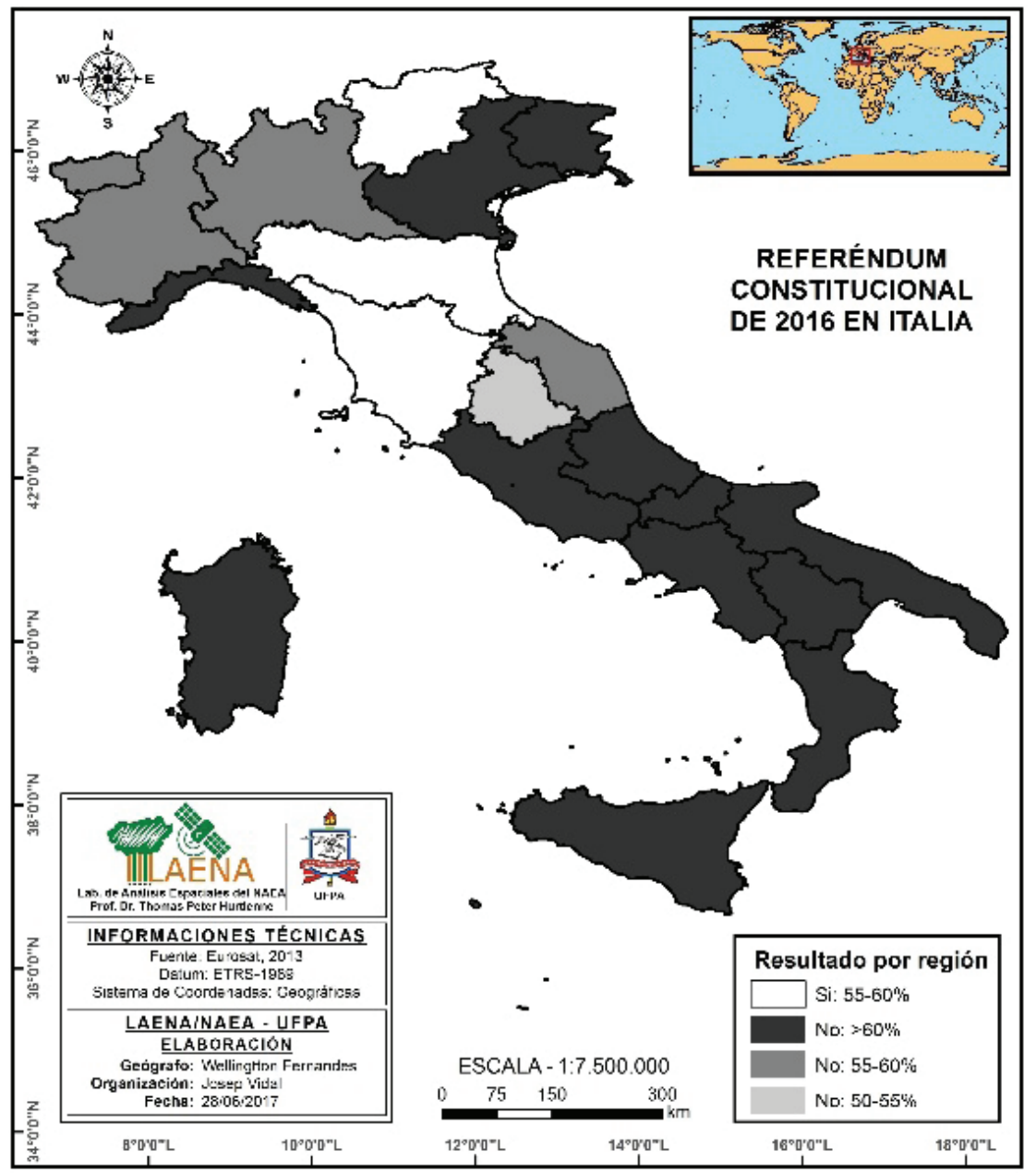

Fuente: autor

Las elecciones generales del año $2014^{26}$ y las elecciones locales que tuvieron lugar en la primavera de 2014 fueron un resultado difícilmente previsible de cualquier analista. En los primeros comicios, Di Virgilio (2015) ya comentó: «Los cambios en la política electoral en las democracias contemporáneas han contribuido a cambiar el enfoque de la investigación de los partidos hacia los candidatos individuales» ${ }^{27}$.

\subsection{Plebiscito secesionista en Brasil (Región Amazónica)}

\subsubsection{Antecendentes}

En Brasil esta consulta está relacionada con la división de un estado, por lo que se limitó en el estado de Pará, cuyo el gobierno se posicionó claramente en contra de aceptar la secesión, trtándose en este caso de un plebiscito (a favor o en contra) en el cual el poder político del estado se manifestó en contra de la opción secesionista ${ }^{28}$. El plebiscito en la Unidad Federativa de Pará (11 de diciembre de 2011, región de la Amazo-

${ }^{26}$ Aldo DI VIRGILIO, et al. Italian general election: evidence from the Italian Candidate Survey.

${ }_{27}$ El politólogo Fortunato Musella analisiza la personaficación en los partidos políticos. En la ultima decada del siglo pasado se caracterizo por el surgimiento de los «partidos personales es uno de los fenómenos políticos más relevantes e innovadores» (Musella, 2014).

${ }^{28}$ No entraremos aquí a valorar la legitimidad de las decisiones con mayorías o de escasas mayorías. En una perspectiva crítica Offe llama la atención sobre los procedimientos: ¿Legitimación a través de la regla de mayoría?, en, Claus OFFE, La gestión política, págs. 163-180. «No puede llegarse a decisiones que sean colectivamente coercitivas si no se ha adoptado una decisión lógicamente previa. Esta se refiere a los procedimientos según los cuales van a adoptarse las decisiones». 
nia oriental) para decidir sobre la división del estado en tres nuevos estados, es un ejercicio de la gobernanza política y su vinculación directa con el territorio. Los motivos y emergencia del movimiento a favor de la secesión sobrepasan argumentos tradicionales como las enormes distancias entre las diferentes capitales y centros administrativos de la región, su renta per cápita, o el grado diferente de desarrollo. El origen parte de iniciativas afines a parlamentarios locales y élites económicas cuyos orígenes históricos se remontan al siglo XIX. El plebiscito fue organizado para decidir sobre una reivindicación histórica sobre la secesión del estado de Pará en tres nuevos estados. Después de un largo proceso jurídico administrativo y político, el Tribunal Superior Electoral (TSE) de Brasilia determinó los términos de la consulta pública que se formalizó en la convocación de un plebiscito ${ }^{29}$. Dos posturas antagónicas aparecen en litigio.

Por un lado, los defensores de la división del estado, recurrieron a antecedentes que tuvieron lugar en otras divisiones de estados ocurridas en Brasil (el estado de Goiás fue desmembrado con la creación del estado de Tocantins en el año 1988) ${ }^{30}$. Ese hecho propició un incremento de las inversiones y un relativo aumento de las condiciones de vida de la población. La división del estado también contribuiría a una mejora en la administración pública y con transferencias económicas federales para una región con bajo IDH. Para las élites locales con intereses en el sector de la minería, agropecuaria e industria maderera, su emancipación significaría la creación de nuevos estados minúsculos estado bajo el control político-económico de estos sectores productivos.

FIGURA 2. SITUACIÓN GEOGRÁFICA DEL ESTADO FEDERAL DE PARÁ Y EVENTUAL CONFIGURACIÓN DE LOS NUEVOS ESTADOS

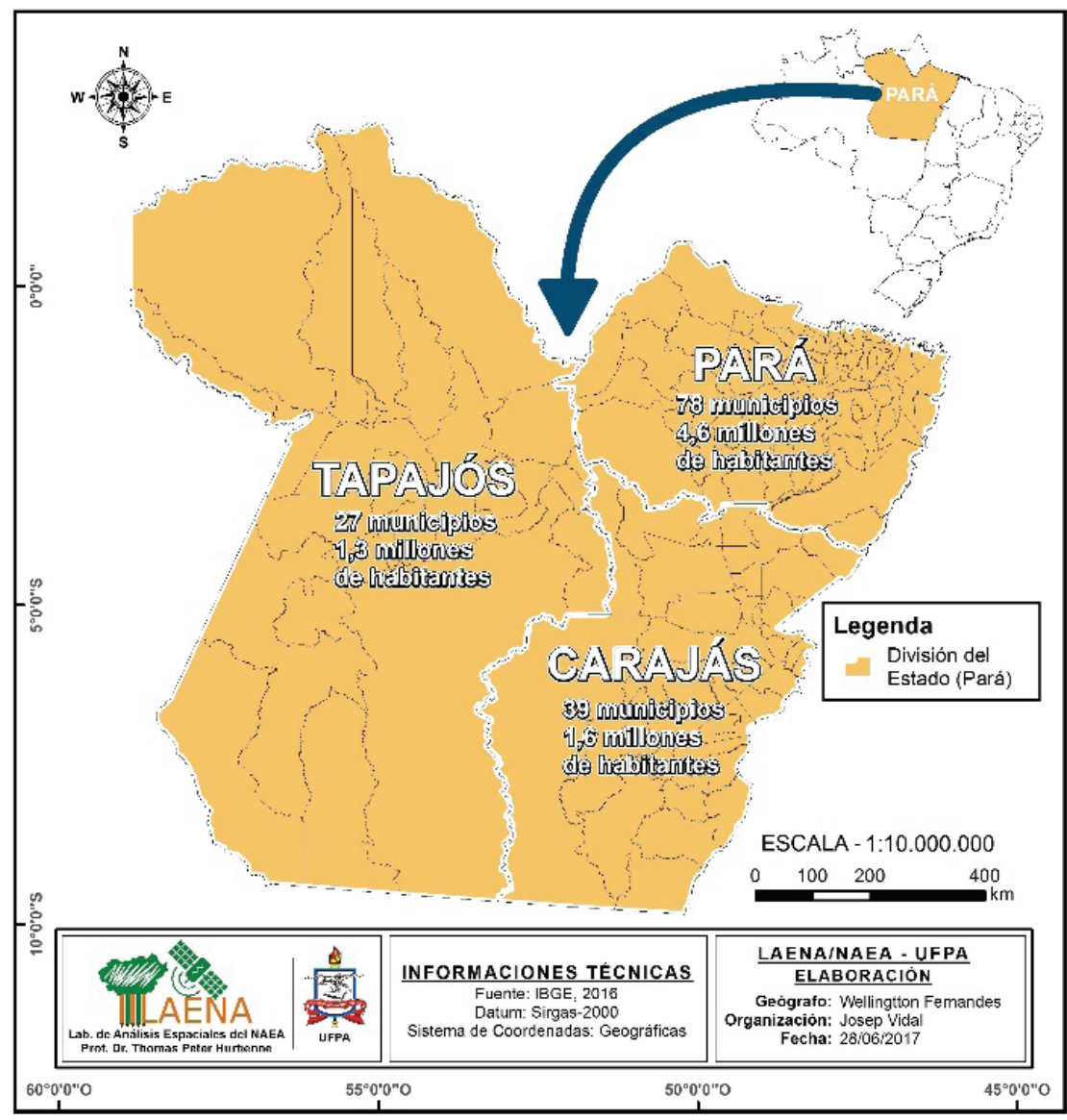

Fuente: autor

29 Para Tapajós, Decreto legislativo núm. 137 de 2011. Diario Oficial da União 3-6-2011. Carajás, Decreto legislativo núm. 136 de 2011. Diario Oficial da União 25-5-2011.

${ }^{30}$ En el año 2013 estistian en Congresso Nacional 13 propuestas para la creación de nuevos estados y territorios. En el caso que que se concreticen significa que Brasil estará formado por 37 estados y 3 territórios con un costo de unos 13.000 de RS (aprox. $6.000 \$, 2011)$. 
Por otro lado, los críticos a la división del estado argumentaron que la división está dirigida tan solo a favorecer las futuras elites políticas de las nuevas regiones formadas, dada la esperada creación de nuevas ofertas de empleo en instituciones públicas, con lo que las esperadas transferencias gubernamentales se canalizarían a otros sectores y se mermarían los beneficios económicos para la población. No menos importante es la drástica reducción de riquezas del estado do Pará ya que con la nueva configuración quedaría prácticamente reducido a un tercio del territorio, con lo que las inversiones necesarias serían insuficientes frente a las demandas necesarias (Figura 2).

Tras un largo debate, se vinculó a dos decisiones formuladas en dos preguntas: una referente a la posibilidad de la división del estado de Pará para la creación de un nuevo estado de Tapajós, y otra para constitución del estado de Carajás. Las preguntas fueron formuladas para toda la población del estado y no solo para los habitantes inscritos en las regiones secesionistas como se pretendía en un principio.

Los apoyos y lobbies empresariales se organizaron en ambas regiones. En la región de Tapajós los grupos locales levantaron grandes expectativas sobre la finalización de grandes obras de infraestructura iniciadas durante el período militar (carretera Transamazónica), después de la creación del estado por decreto. El movimiento emancipador se intensifico con la creación de un Comité de alcaldes de la región en el año 1991, el cual realizó los trámites administrativos al Congreso Nacional para solicitar la consulta.

La creación del estado de Carajás tiene sus inicios en la década de los años 1980 siendo motivado es especial por el desarrollo económico de la región (descubrimiento casual de la mayor reserva de bauxita y de hierro del planeta), la mina de oro en Sierra Pelada.

\subsubsection{Resultados}

Los resultados del plebiscito fueron contrarios a la división del estado en función de las preguntas: «¿Usted está a favor de la división del estado de Pará para la creación del estado de Carajás?», y «¿Usted está a favor de la división del estado de Pará para la creación del estado de Tapajós?» (Cuadro 4).

CUADRO 4. RESULTADO dEL PLEBiscito SEgúN LA REgión

\begin{tabular}{|c|c|c|c|}
\hline & $\begin{array}{c}\text { Si } \\
\text { En las regiones } \\
\text { secesionistas }\end{array}$ & $\begin{array}{c}\text { No } \\
\text { Estado (regiones } \\
\text { no secesionistas) }\end{array}$ & $\begin{array}{c}\text { No } \\
\text { Belém, } \\
\text { capital del estado }\end{array}$ \\
\hline $\begin{array}{l}\text { A favor de la división del estado } \\
\text { y para creación del estado de Carajás }\end{array}$ & $\begin{array}{c}\text { Carajás } \\
98.63 \% \\
\text { de los votos válidos }\end{array}$ & $\begin{array}{c}66.60 \% \\
\text { de los votos válidos }\end{array}$ & $\begin{array}{c}94.87 \% \\
\text { de los votos válidos }\end{array}$ \\
\hline $\begin{array}{l}\text { A favor de la división del estado } \\
\text { y para la creación del estado de Tapajós }\end{array}$ & $\begin{array}{c}\text { Tapajós } \\
94.88 \% \\
\text { de los votos válidos }\end{array}$ & $\begin{array}{c}66.08 \% \\
\text { de los votos válidos }\end{array}$ & $\begin{array}{c}93.88 \% \\
\text { de los votos válidos }\end{array}$ \\
\hline
\end{tabular}

Fuente: Tribunal eleitoral Estado do Pará

El voto se repartió de diferente forma y varió según la región optase o no a la secesión. En la capital del estado (Belém) con población contraria a la secesión el «no» a la creación del estado de Tapajós alcanzó el $93,88 \%$ de los votos y el «no» a la creación del estado de Carajás fue de 94,87\%. En las posibles capitales de los nuevos estados fue diferente con un masivo apoyo. En la ciudad de Santarém (candidata a capital del estado), $97.78 \%$ de los electores que participaron a las urnas voto a favor de la creación de Carajás e $98.63 \%$ a favor da creación de Tapajós. Mientras que en el municipio de Marabá (capital candidata del estado), $93,26 \%$ de los votos fueran favorables a la creación de Carajás e $92.93 \%$ a favor de la creación de Tapajós. El índice de participación general fue del $74.29 \%$.

\subsubsection{Análisis sistémico}

Presentamos también unas hipótesis que pueden sintetizarse en:

- Primera. El «factor de cohesión» de la política ha puesto de relieve una doble posibilidad. Mientras que la posición gubernamental ha imperado en la parte central politico-administrativa del estado, 
GAPP. Nueva Época - N. 18, noviembre 2017 - ISSN: 1989-8991 - DOI: 10.24965/gapp.v0i18.10383 - [Págs. 62-80]

Referéndum constitucional en Italia y plebiscito territorial en Brasil. Un enfoque de sistémico de gobernanza

Josep Pont Vidal

en las regiones secesionistas ha imperado el discurso de las élites políticas y económicas para la creación de los nuevos estados. No significa una posibilidad de autoorganización de la sociedad (de los sistemas y subsistemas) puesto que la creación de nuevos estados significación una repetición a pesqueña escala de los sisemas y subsistemas existentes. En Brasil y en la región de la Amazonia en particular, las estructuras del Estado de configuran de forma funcionalmente acéntrica (Neves, 2004) o sea, con una escasa existencia de centros decisorios y administrativos que cumplan las funciones de centralidad (administrativa y política). La circularidad intersistémica (vinculación comunicativa entre los sistemas) antrópica es baja, manifestada en el sistema económico y la elevada tasa de informalidad laboral en el estado que repercute en la capacidad de actores y de los sindicatos en influenciar en el sistema político-administrativo ${ }^{31}$. El proceso tiene lugar mediante un acoplamiento estructural insuficiente de cada uno de los subsistemas que componen la sociedad (Cuadro 5).

CUADRO 5. SISTEMAS PRINCIPALES, OPERACIONES Y CAMBIOS FUNCIONALES (ESTADO DE PARÁ)

\begin{tabular}{llc}
\hline & Cambios funcionales y operaciones cuantificables & $\begin{array}{c}\text { Abertura } \\
\text { opearativa }\end{array}$ \\
\hline $\begin{array}{l}\text { Sistema } \\
\text { económico }\end{array}$ & $\begin{array}{l}\text { Apertura a la globalización. Tasa de informalidad laboral: } 67.4 \% \\
\text { entre los hombres, 67.7 entre las mujeres* } \\
\text { Reforma posesión tierras** }\end{array}$ & $\begin{array}{c}\text { Baja } \\
\text { Insuficiente }\end{array}$ \\
\hline \multirow{2}{*}{ Sistema educativo } & $\begin{array}{l}\text { 2017. Reestructuración orientada a la vida profesional. 54\% de los adultos } \\
\text { con edad entre 25 y 64 años no han completado la enseñanza media (Brasil, }\end{array}$ & Baja \\
\hline Sistema científico & Impulso cooperación público-privada (universidades-empresas) & Baja \\
\hline Sistema jurídico & $\begin{array}{l}\text { Gobierno abierto y Justicia abierta } \\
\text { Defensoría Pública: actor entre gestión y transformación }\end{array}$ & Insuficiente \\
& Baja \\
\hline \multirow{2}{*}{ Sistema religioso } & $\begin{array}{l}\text { Sistema base de conexiones intersistémicas } \\
\text { Declaran «sin religión»: entre } 6.72 \text { y } 6.69 \% * * *\end{array}$ & Alta \\
\hline
\end{tabular}

* Instituto Brasileiro de Geografia e Estatística (IBGE, 2015).

** http://imazon.org.br/PDFimazon/Portugues/livros/REG_FUNDPARA_WEB.pdf.

*** https://www.oecd.org/brazil/Education-at-a-glance-2015-Brazil-in-Portuguese.pdf.

**** CPS/FGV a partir de microdatos de POF, 2009.

Fuente: autor

- Segunda. La opcion para la secesión ha sido intepretada como una posibilidad de refuerzo del sistema político-administrativos de los posibles nuevos estados, con la posibilidad de creación de nuevas estructuras político-administrativas (repetidoras de las ya existentes) y formación de nuevas élites políticas. Las observaciones y propuestas de cambios funcionales de los diversos sistemas parciales de la sociedad paraense ha sido promovidas por el gobierno federal y estadual mediante planos de desarrollo (sostenible y particpativo) aunque con la aplicación de conceptos que han limitado conceptualmente las observaciones de diferenciación funcional (Cuadro 6).

CUADRO 6. PLANOS DE DESARROLLO (REGIONAL) SOSTENIBLE Y CONCEPTOS DOMINANTES

\begin{tabular}{|c|c|c|c|c|}
\hline Planos & PDE & Tucurui & $\begin{array}{l}\text { PDSBM Belo } \\
\text { Monte e PDST }\end{array}$ & $\begin{array}{l}\text { PDRS*: Marajo, Xingu, } \\
\text { Transamazônica, PPDJUS, } \\
\text { Tocantins, PDRS Tucuruí }\end{array}$ \\
\hline $\begin{array}{l}\text { Fundamento } \\
\text { teórico }\end{array}$ & $\begin{array}{l}\text { Sistémico } \\
\text { (sistemas } \\
\text { abiertos) }\end{array}$ & $\begin{array}{l}\text { Sistémico (sistemas } \\
\text { abiertos) } \\
\text { Tecnocrático }\end{array}$ & $\begin{array}{l}\text { Análisis de } \\
\text { politicas }\end{array}$ & $\begin{array}{l}\text { Análisis de políticas } \\
\text { Teoría de la gobernanza } \\
\text { Policy Science }\end{array}$ \\
\hline
\end{tabular}

31 Datos según IBGE y el Ministerio das Cidades. 


\begin{tabular}{lllll}
\hline $\begin{array}{l}\text { Conceptos } \\
\text { clave }\end{array}$ & $\begin{array}{l}\text { Jerarquia } \\
\text { Sistemas } \\
\text { Desarrollo } \\
\text { técnico }\end{array}$ & $\begin{array}{l}\text { Desarrollo local } \\
\text { Innovación tecnologica } \\
\text {-oportunidades }- \\
\text { amenazas }\end{array}$ & $\begin{array}{l}\text { Dinamismo } \\
\text { Sostenibilidad } \\
\text { Modernización } \\
\text { Competitividad } \\
\text { regional }\end{array}$ & $\begin{array}{l}\text { Gobernanza } \\
\text { Institucionalismo, orientado a la } \\
\text { gestión de los actores y del territorio }\end{array}$ \\
\hline Referencias & $\begin{array}{l}\text { Economicismo } \\
\text { clásico }\end{array}$ & $\begin{array}{l}\text { Metodologia de } \\
\text { planificación }\end{array}$ & Limitada & $\begin{array}{l}\text { Extensa } \\
\text { Conservadora-progresista }\end{array}$ \\
\hline
\end{tabular}

* Planes de Desarrollo Regional Sostenible.

En los planos destacaba un cambio de paradigmas en las propuestas de planificación, al pasar de los modelos iniciales todavía fundamentados en el concepto «sistémico-burocrático» (sistemas abiertos) hacia paradigmas basados en las teorías de la gobernanza y en la gestión de los actores (públicos, privados y sociales).

Los estudios empíricos disponibles manifiestan que las instituciones gubernamentales han aplicado modelos participativos y dinámicos, aunque fundamentados en la conceptción de la «doble diferenciación» (explotadores/explotados, o clase A,B,C) ignorándose otras posibilidades.

- Tercera. Las regiones secesionistas. La fractura política y social profunda, entre regiones va también más allá de las opciones políticas (y del eje clásico izquierda-derecha). Sobrepasa los parámetros clásicos y plantea profundos problemas y concepciones a la gobernanza política y a la gobernanza en general. (diferenciación tridimensional).

Los movimientos sociopolíticos y acciones jurídicas impulsoras del plebiscito pueden ser interpretados como una posibilidad de autoorganización de la sociedad en los ámbitos político y administrativo y territorial. Esta posibilidad no surge de las dinámicas sistémicas territoriales sino que está direccionada «desde arriba», impulsada por las operaciones llevadas a cabo por las élites económicas y políticas con el objetivo de mantener y aumentar su control directo (y privilegios) y a su vez mantener una cierta distancia del control jurídico y político del Estado de derecho brasileño.

\subsubsection{Unas conclusiones}

La teoría de sistemas asume la doble pretensión central de ser una teoría para la teoría y también una teoría para la práctica.

La idea de gobernanza sistémica no consiste en crear en un plano teórico un sistema con operaciones autorreferenciales, o una nueva estructura burocrática o administrativa, sino que se trata de un modelo teórico (y por lo tanto abstracto) que dispone de la capacidad operativa de seleccionar las comunicaciones y los códigos procedentes de los otros sistemas que conforman la sociedad y de la acción individual. El sistema político asume un papel de coordinador de las comunicaciones procedentes de los otros subsistemas. Las comunicaciones procedentes de otros sistemas y surgidas de estos son filtradas, seleccionadas e interpretadas por cada subsistema en un lenguaje de codificación común.

El territorio ha de entenderse como el espacio en el que actúan los subsistemas (entorno), y se define como sistema global diferenciado por las comunicaciones y códigos que emanan de los sistemas parciales. El entorno interno de este sistema está constituido por el entorno político-administrativo en el que se insieren los códigos lingüísticos y culturales. Aquí las lógicas de direccionamiento y de poder se reflejan a escala territorial, con lo que la legitimidad de las decisiones mayoritarias también deben ser reanalizadas ${ }^{32}$.

El introducir el plano subjetivo representa la posibilidad de aportar las informaciones necesarias para la comprensión de la acción y del impacto en los respectivos subsistemas en el plano de la toma de decisiones individual. Permite disponer de informaciones sobre las preferencias y actitudes respecto a las decisiones y acciones colectivas influenciadas por los procesos (sistémicos) pero también por la capacidad de pensamiento en un sentido basada en la interacción. El debate sobre el meta direccionamiento de la sociedad reafirma la democracia como modelo de dirección de las sociedades complejas, aunque no debería olvidar

${ }^{32}$ En una perspectiva crítica Claus Offe (1984) llama la atención sobre los problemas, procedimientos y legitimidad de las decisiones mayoritarias. 
GAPP. Nueva Época - N. 18, noviembre 2017 - ISSN: 1989-8991 - DOI: 10.24965/gapp.v0i18.10383 - [Págs. 62-80]

Referéndum constitucional en Italia y plebiscito territorial en Brasil. Un enfoque de sistémico de gobernanza

Josep Pont Vidal

el interrogante sobre si la idea de democracia es el único modelo posible de organización de sistemas complejos o si puede existir otro modelo.

Las consultas en Italia y Brasil ponen de manifiesto las limitaciones de los conceptos en que se ha apoyado la gobernanza en su extensión política y territorial. Las limitaciones y la incapacidad del sistema político aparecen en la forma de prevenir y de ofrecer las respuestas necesarias (endógeno) y en sus capacidades de gestión, como las que se derivan de un entorno con cambios sociales y estructurales funcionales no percibidos previamente (exógenos). La posibilidad de autoorganización de la sociedad y de los sistemas, se han manifestado «hacia dentro», con un acoplamiento estructural insuficiente de cada sistema (Cuadro 7).

CUADRO 7. DIFERENCIAS SISTÉMICAS EN LOS DOS CASOS EXPUESTOS

\begin{tabular}{lll}
\hline & Italia & $\begin{array}{l}\text { Estado Pará } \\
\text { Región amazonia brasileña }\end{array}$ \\
\hline Estructuras del estado & Funcionalmente policéntrica & Funcionalmente acéntrica \\
\hline «meta contribución» del sistema político & $\begin{array}{l}\text { «hacia fuera» sobrepasa los } \\
\text { intereses del partido gubernamental }\end{array}$ & $\begin{array}{l}\text { Doble «hacia fuera/dentro» } \\
\text { dependiendo de la región }\end{array}$ \\
\hline $\begin{array}{l}\text { Impacto en el sistema político } \\
\text { Circularidad antrópica } \\
\text { (en la perspectiva de los partidos } \\
\text { políticos) }\end{array}$ & Elevada abstención (no deseada) & $\begin{array}{l}\text { Resultado deseado político } \\
\text { (participación obligatoria) }\end{array}$ \\
\hline $\begin{array}{l}\text { Autoorganización de la sociedad } \\
\text { Interconexión sistémica }\end{array}$ & Elevado acoplamiento & Bajo acoplamiento estructural \\
\hline $\begin{array}{l}\text { Diferenciación funcional } \\
\text { (en la perspectiva de los partidos } \\
\text { políticos) }\end{array}$ & En caso del referéndum: alta & En caso del plebiscito: alta \\
\hline
\end{tabular}

Fuente: elaboración propia

En Italia, el triunfo del «no» a su propuesta (ha supuesto y la renuncia al cargo siendo substituido por el nuevo Primer ministro Paolo Gentilone). El sistema político del Brasil ha vivido en el año 2016 el impeachment histórico ${ }^{33}$ de la Presidente Dilma Rousseff solo los cuales no entraremos en detalle aquí (a partir de argumentos cuestionables y construidos sobre su gestión y decisiones en el ámbito económico) asumiendo a presidencia en el año 2016, el hasta entonces Vice-presidente Michel Temer.

Por último, indicar que en ninguno de los dos casos tiene lugar una sotuacion de «doble contingencia» (expectativas respecto al otro actor), puesto que las dos formas de consulta fueron impulsadas por el sistema político.

\section{REFERENCIAS BIBLIOGRÁFICAS}

ABBOTT, K. W. y SNIDAL, D. (2009), "The governance triangle: Regulatory standards institutions and the shadow of the state", en The Politics of Global Regulation, págs. 44-88. Princeton University Press.

AGUILAR, L. F. (2009), Gobernanza: normalización del concepto y nuevas cuestiones. Barcelona: Esade.

ARENILLA. M. (2015), Ciudad, gobernanza y planificación estratégica. Madrid: Dykinson.

BANG, H. (2003), Governance as social and political communication. Manchester: Manchester University Press.

BATTINI, S. y FIORENTINO, L. (2014), "Venti anni di 'politica e amministrazione in Italia', Istituto di Ricerche sulla Pubblica Amministrazione", IRPA Working Paper, Policy Papers Series, núm. 1/2014.

BENZ, A. (2004), "Mulitlevel Governance. Governance im Mehrebenensystem", en A. BENZ, A. (Hrsg.), Governance. Regieren in komplexen Regelsystemen. Eine Einführung, págs. 125-146. Wiesbaden.

BERGER, P. y LUCKMANN, Th. (2003), La construcción social de la realidad. Buenos Aires: Amorrortu.

${ }^{33}$ Debemos recordar el primer impeachment fue de Fernando Collor de Mello en el año 1992. 
GAPP. Nueva Época - N. 18, noviembre 2017 - ISSN: 1989-8991 - DOI: 10.24965/gapp.v0i18.10383 - [Págs. 62-80]

Referéndum constitucional en Italia y plebiscito territorial en Brasil. Un enfoque de sistémico de gobernanza

Josep Pont Vidal

BEUNEN, R; VAN ASSCHE, K.; Duineveld, M. (2015), Evolutionary Governance Theory: theory and applications. Heidelberg: Springer.

BEYERLE, M. (1994), Staattheorie und Autopoiesis. Über die Auflösung der modernen Staatsidee im nachmodernen Denken durch die Theorie autopoietischer Systeme und der Entwurf eines nachmodernes Staatskonzepts. Frankfurt, a.M.: Peter Lang.

CAFFANI, F. y PISTOR, K. (2014), "Regulatory capabilities: A normative framework for assessing the distributional effects of regulation", Regulation \& Governance, 9 (2): 95-107, DOI: 10.1111/rego.12065.

CERRILLO, A. (2005), "La gobernanza hoy: introducción”, en A. CERRILLO, 10 Textos de referencia, págs. 11-36. Madrid: Instituto Nacional de Administración Pública.

CHEIBUB, A.; PRZEWORSKI, A. y SAIEGH, S. (2002), "Governos de Coalizão nas Democracias Presidencialistas e Parlamentaristas", Dados, núm. 45. DOI: 10.1590/s0011-52582002000200001.

CASADIO, G. y CAGLIARDI, G. (2016), "Referendum costituzionale, minoranza Pd non firma. Renzi: "C'è una parte che si oppone a tutto", La Repubblica, 20 aprile, 2016.

CERRI M. y SOLI V. (2009), I mestieri del sindacalista. Tra rappresentazione soggettiva e ridefinizione professionale. Roma: Ediesse.

DI VIRGILIO, A. et al. (2015), Italian general election: evidence from the Italian Candidate Survey, DOI: https://doi. org/10.1017/ipo.2015.15.

DRUWE, U. (1989), "Rekonstruktion der 'Theorie der Autopoiese' als Gesellschafts und Steuerungsmodell”, en A. GÖRTLIZ (Hrg.) Politische Steuerung sozialer Systeme. Mediales recht als politisches Steuerungskonzept. Pfaffenweiler.

DUIT, A. y GALAZ, V. (2008), "Governance and Complexity. Emerging Issues for Governance Theory", Governance, 21 (3): 311-335. DOI: 10.1111/j.1468-0491.2008.00402.x.

GAWRON, Th. (2010), Steuerungstheorie, Policy-Forschung und Governance-Ansatz: zum verfehlten GovernanceKonzept der Regionalforschung. Leipzig: Forschungsverbund KoReMi.

GOBIERNO BRASIL (2011), Decreto legislativo núm. 137 de 2011, Diario Oficial da União 3-6-2011.

GOBIERNO BRASIL (2011), Decreto legislativo núm. 136 de 2011. Diario Oficial da União 25-5-2011.

GONNET, J. P. (2010), "La política desde la teoría de sistemas sociales de Niklas Luhmann". Argumentos, (64): 303-310.

GÖRLITZ, A. y BURTH, H. P. (1998), Politische Steuerung. Ein Studienbuch. Opladen.

HELLMANN, K.-U.; FISCHER, K. y BLUHM, H. (Hrsg.)(2003), Das System der Politik. Berlín: Springer.

HOLZ, K. (2003), "Politik und Staat. Differenzierungtheoretische Probleme in Niklas Luhmann Theorie des politischen Systema”, en K.-U., HELLMANN; K. FISCHER; H. BLUHM, (Hrsg), Das System der Politik, págs. 34-48. Berlín: Springer.

INSTITUTO DE RELACIONES INTERNACIONALES. Universidad Nacional de La Plata (2016), Disponible en: http:// www.iri.edu.ar/wp-content/uploads/2016/12/referendum-constitucional-italia-francesca-staiano.pdf.

ITALIA (2016), "La Riforma Costituzionale". Testo di legge costituzionale pubblicato sulla Gazzetta Uciale, núm. 88 del 15 aprile 2016, Testo a fronte con la Costituzione vigente. Disponible en: http://www.altalex.com/documents/ leggi/2016/04/13/riforma-costituzionale-il-testo.

KLAWITTER, J. (1992), "Staatstheorie als Steuerungstheorie? Vorüberlegungen im Hinblick auf ein staatstheoretisch fundiertes, akteurzentriertes und verhandlungsorientiertes Netzwerk polyzentrischer Gesellschaften", en: H. BUßHOFF (Hg.), Politische Steuerung. Steuerbarkeit und Steuerungsfähigkeit. Baden-Baden: Nomos, pág. 193-239.

KOOIMAN, J. (2003), Governing as governance. London: Sage. DOI: 10.4135/9781446215012.

KOOIMAN, J. y BAVINK, M., CHUENPAGDEE, R., MAHON, R.; PULLIN, R. (2008), "Interactive Governance and Governability: An Introduction. Journal of Transdisciplinary", Environmental Studies, 7 (1), 1-11. Disponible en: http://dare.uva.nl/document/2/59200.

KOOIMAN, J. y VAN FLIET, M. (2000), "Self-Governance as a mode of Societal-Governance", Public Management, 2, 3 (19), 359-377. DOI: https://doi.org/10.1080/14616670050123279.

LA VALLE, R. (2016) “Italia: la verdad sobre el referendum de Renzi”, Sin Permiso. Disponible en: http://www. sinpermiso.info/textos/italia-la-verdad-sobre-el-referendum-de-renzi.

LANGE, S. y BRAUM, D. (2000), Politische Steuerung zwischen System und Akteur. Opladen: Westdeutscher. DOI:10.1007/978-3-663-11096-5.

LEAT, D.; SETZLER, K. et al. (2002), Towards holistic governance: the new reform agenda. Basingstoke: Palgrave. DOI: $10.1093 /$ parlij/gsg026.

LEYDESDORFF, L. (2015), Una teoría sociológica de la comunicación. La autoorganización de la sociedad basada en el conocimiento. México: Universidad Iberoamericana.

LUHMANN, N. (1981), Politische Theorie im Wohlfahrtsstaat. München/Wien: Olzog.

- (1981a), "Theoretische Orientierung der Politik", en N. LUHMANN (Hrsg.), Soziologische Aufklärung 3. Soziales System, Gesellschaft, Organisation, págs. 287-293. Opladen: Westdeutcher Verlag. DOI: 10.1007/978-3-66301340-2_16.

- (1989), "Politische Steuerung: Ein Diskussionsbeitrag", Politische Vierteljahresschrift, 30 (1), 4-9.

- (1988), "Selbsreferentielle Systeme", en F. B. SIMON (Org.) Lebende Systeme: Wirklichkeitskonstruktionen in der systemischen Therapie, págs. 47-53, Berlin. 
GAPP. Nueva Época - N. 18, noviembre 2017 - ISSN: 1989-8991 - DOI: 10.24965/gapp.v0i18.10383 - [Págs. 62-80]

Referéndum constitucional en Italia y plebiscito territorial en Brasil. Un enfoque de sistémico de gobernanza

Josep Pont Vidal

MANNA, P. (2010). Network Governance in Three Policy Areas with Implications for the Common Core State Standards Initiative. Washington: Thomas $\mathrm{B}$. Fordham Institute.

MATURANA, H. y VALERA, F. (2003), El árbol del conocimiento. Las bases biológicas del entendimiento humano. Buenos Aires: Lumen.

MAYNTZ, R. (2009), Über Governance. Institutionen und Prozesse politischer Regelung. Schriften aus dem MaxPlanck-Institut für Gesellschaftsforschung, Bd. 62. Frankfurt a.M.: Campus.

- (2005), "Governance Theorie als fortentwickelte Steuerungstheorie?”, en F. G. SCHUPPERT (Hrsg.), GovernanceForschung, págs. 11-20. Baden-Baden.

- (2001), "Zur Selektivität der steuerungstheoretischen Perspektive", en H. P., BURTH, y A. GÖRLITZ (Hrsg.), Politische Steuerung in Theorie und Praxis, págs. 7-27. Baden-Baden.

MAYNTZ, R. y SCHARPF, F. W. (Hg.) (1995), Steuerung und Selbsorganization in staatnahen Sektoren en R. MAYNTZ; F. W. SCHARPF (Eds.) Gesellschaftliche Selbsregelung und politische Steuerung, págs. 9-38. Frankfurt a.Main.: Campus.

MUSELLA, F. (2014), How personal parties change: party organisation and (in)discipline in Italy (1994-2013), págs. 222-237. DOI: http://dx.doi.org/10.1080/23248823.2014.969005.

NETO, O. A. (2006), Presidencialismo e Governabilidade nas Américas. Rio de Janeiro: FGV.

NEVES, M. (2004), "Vom Rechtspluralismus zum sozialen Durcheinander: der Mangel an Identität der Rechtssphäre(n) in der peripheren Moderne und seine Implikationen in Lateinamerika", en BRUNKHORST, H.; MATIASKE, W. (org.), Zentrum und Peripherie. Mering: Hammp, 2004. págs. 165-194.

OFFE, C. (1984), "Politische legitimation durch Mehrheitsentscheidung?", en B. GUGGENBERGER; C. OFFE (Hrsg.), An den Grenzen der Mehrheitsdemokratie, págs. 150-183. Opladen: VS Verlag.

OSBORNE, S. (2006), “The New Public Governance?”, Public Management Review, 8(3): 377-387, DOI: 10.1080/14719030600853022. Disponible en: http://spp.xmu.edu.cn/wp-content/uploads/2013/12/the-New-PublicGovernance.pdf.

PASQUINO, G. (2015), La Costituzione in trenta lezioni. Utet.

PRATS. J. (2005), "Modos de gobernación de las sociedades actuales", en A. CERRILLO (coord.), La gobernanza hoy: 10 textos de referencia. Madrid: INAP.

POBLETE, M. (2011), "Gobernanza y diferenciación funcional en órdenes sociales complejos. Una aproximación al caso Latinoamericano", Revista Ciencias Sociales, 26: 25-37.

PONT, J. (2015), "Formação institucional e Desenvolvimento regional no Estado do Pará", en DA SILVA, F. C., Formação Institucional da Amazônia. Belém: NAEA, págs. 347-391.

- (2017), La innovación en la gestión pública. Madrid: Ediciones La Catarata.

- (2015), "Autopoiesis, autoorganización y cierre operativo en las organizaciones desde la perspectiva postestructuralista", International Journal of Organizations / Revista Internacional de Organizaciones, Issue 14, págs. 31-55. Disponible en: http://www.revista-rio.org/index.php/revista_rio.

REGALIA I. (2009), Quale rappresentanza. Dinamiche e prospettive del sindacato in Italia. ISF/Ediesse.

SCHIMANK, U. y GLAGOW, M. (1984), "Formen politischer Steuerung: Etatismus, Subsidiarität, Delegation und Neokorporativismus”, en M. GLAGOW (Hrsg.), Gesellschaftssteuerung zwischen Korporatismus und Subsidiarität, págs. 4-28. Bielefeld: AJZ.

SCHWINN, T. et al. (2011), Soziale Differenzierung. Handlungstheoretische Zugänge in der Diskussion. Wiesbaden: VS Verlag.

SKELCHER, C. (2005), "The Public Governance of Collaborative Spaces: Discourse, Design and Democracy", Public Administration, 83 (3): 573-596. DOI: 10.1111/j.0033-3298.2005.00463.x.

STICHWEH, R. (2001), "Niklas Luhmann (1927-98)", en N. J. SMELSER; P. B. BALTES (eds.), International Encyclopedia of the Social and Behavioral Sciences. Oxford, págs. 9.097-9.102. Disponible en: http://iva.dk/jni/ lifeboat/info. asp?subjectid=243.

SORENSEN, E. y TRIANTAFILLOU, P. (2009), The Politics of Self-Governance. London / New York: Routledge. DOI: $10.4324 / 9781315554259$.

SUBIRATS, J.; PARÉS, M. y BLANCO, I. (2009), "Calidad democrática y redes de gobernanza: evaluar la participación desde el Análisis de Políticas Públicas”, en M. PARÉS (coord.), Participación y calidad democrática: evaluando nuevas formas de democracia participativa. Barcelona: Ariel.

TÜRKE, R. E. (2008), Governance: Systemic Foundation and Framework. Heidelberg: Physica Verlag.

VALLESPÍN, F. (1993), "Introducción", en N. LUHMANN, Teoría política en el estado de Bienestar, págs. 9-29. Madrid: Alianza.

VON HALDENWANG, Ch. (2005), Systemic gobernance and development in Latin America. Review 85, Santiago de Chile: Cepal.

WILLKE, H. (2014), Systemtheorie III: Steuerungstheorie. Konstanz: UKV.

- (2006), Global governance. Bielefeld: Transkrip.

- (1992), Ironie des Staates. Frankfurt a.M.: Suhrkamp.

- (1992a), Entzauberung des Staates. Überlegungen zu einer gesellschaftlichen Steuerungstheorie. Frankfurt a. M.: Suhrkamp. 